\title{
Laser-Induced Fluorescence Decay of 2-Methyl-, 2-Methoxy-, and 2-Ethylnaphthlene on $\alpha$-Alumina during Temperature Programmed Desorption
}

\author{
Bradly B. Baer, ${ }^{1,2}$ Shanan Lau, ${ }^{1}$ Hannah E. Ryan, ${ }^{1}$ K. A. Martin, ${ }^{1,2}$ and A. M. Nishimura ${ }^{1}$ \\ ${ }^{1}$ Department of Chemistry, Westmont College, Santa Barbara, CA 93108, USA \\ ${ }^{2}$ Department of Chemistry, Point Loma Nazarene University, San Diego, CA 92106, USA
}

Correspondence should be addressed to A. M. Nishimura; nishimu@westmont.edu

Received 23 June 2012; Accepted 30 July 2012

Academic Editor: Djordje Mandrino

Copyright (C) 2013 Bradly B. Baer et al. This is an open access article distributed under the Creative Commons Attribution License, which permits unrestricted use, distribution, and reproduction in any medium, provided the original work is properly cited.

\begin{abstract}
The decay of electronically excited molecular films of 2-methylnaphthalene (2-MN), 2-methoxynaphthalene (2-MeON), and 2ethylnaphthalene (2-EN) on a crystal of $\alpha$-alumina was monitored as a function of temperature with temperature programmed desorption (TPD) experiments. By assuming an exponential decay, the rate constants of the relaxation to the ground state were observed to have two components $( \pm 20 \%)$ by laser induced fluorescence (LIF). For the 2-MeON, 2-MN, and 2-EN excimer, the longer components were 35,25 , and $23 \times 10^{6} \mathrm{~s}^{-1}$, respectively. Rate constants for the trap fluorescence for 2-MeON, 2-MN, and 2-EN were 100,44 , and $23 \times 10^{6} \mathrm{~s}^{-1}$, respectively. In separate experiments, the effect of a molecule that does not fluoresce and has a lower desorption temperature than the fluorophores was studied by deposition of a bilayer. 1-Chlorohexane (1- $\mathrm{CH})$ was chosen as the second layer to the fluorophore and the results gave clues to the complexity of the surface dynamics that occur as the surface is heated. For these bilayer systems, a second excimer formed during the TPD subsequent to the desorption of 1- $\mathrm{CH}$, and their rates are given in parenthesis: for $2-\mathrm{MeON}, 2-\mathrm{MN}$, and $2-\mathrm{EN}$, the long components were $30(36), 25(45)$, and $23(42) \times 10^{6} \mathrm{~s}^{-1}$, respectively.
\end{abstract}

\section{Introduction}

The surface dynamics of vapor deposited organic fluorophores can be observed by taking advantage of the sensitivity of the luminescence intensity and the relaxation rate constants to the molecular environment as the surface is heated. Vapor deposition of volatile organic molecules onto surfaces prepares them amorphously [1]. Excimers form when these molecules are electronically excited because of the proximity between molecules, which is less than the critical distance of $3.0 \AA$ [2]. As the surface is heated, the adlayer molecules can undergo disorder-to-order transition, whereupon the intermolecular distance is larger than the critical distance for excimer formation, and molecular fluorescence similar to that observed in solution is observed. Hence, during a TPD experiment, both the spectra and fluorescence decay can be monitored to indirectly observe molecular rearrangements, so that a model of the surface dynamics can be speculated.
The excimers of naphthalene and substituted naphthalenes have been previously characterized [3] and their radiative decay rate constant is about $0.17 \times 10^{6} \mathrm{~s}^{-1}$ [4]. The rate constant for the excimer decay of naphthalene in solution has been reported to be $3.3 \times 10^{6} \mathrm{~s}^{-1}$ [5]. The rates constants for methylnaphthalene in ethanol are $\sim 5 \times 10^{6} \mathrm{~s}^{-1}$ [6], for dimethylnaphthalene $1.4 \times 10^{6} \mathrm{~s}^{-1}$ in $\mathrm{n}$-heptane, and for the pure liquid $2.2 \times 10^{6} \mathrm{~s}^{-1}$ [7], whereas the monomer in dilute $\mathrm{n}$-hexane, the rate is larger, $5 \times 10^{6} \mathrm{~s}^{-1}$ [7]. Others have given rate constants for 1-MN in ethanol for the monomer and excimer as 24 and $3.3 \times 10^{6} \mathrm{~s}^{-1}$, respectively [2].

The vacuum deposited fluorescence of naphthalene was reported by Nakayama et al. [1]. We first reported the wavelength-resolved TPD of several naphthalene molecules [8]. We have extended that study to other naphthalene-like molecules, in particular 2-MeON, 2-MN, and 2-EN and in this paper, we report their rate constants. In addition, 1-CH 


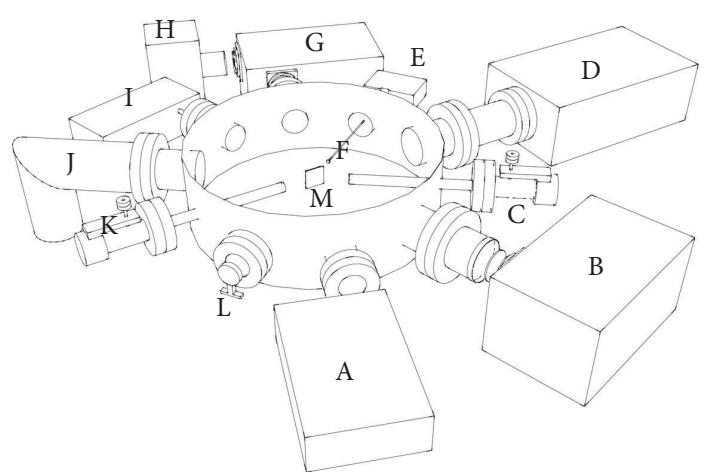

FIGURE 1: Experimental setup. A: Quadrupled Nd: YAG laser, B: focusing lens and monochromator with PMT, leak valve with collimator, C: leak value, D: quadrupole mass spectrometer, E: spectrometer, F: fiber optic cable and focusing lens, $\mathrm{H}$ : high pressure Hg lamp, I: ion gauge, J: to turbo pumps, K: leak valve with 1chlorohexane, to turbo pumps, L: PC camera, $\mathrm{M}: \mathrm{Al}_{2} \mathrm{O}_{3}$ (0001) crystal.

was deposited as a bilayer with the fluorophore to observe the spectral effect of this molecule during the TPD. 1-CH desorbs at $200 \mathrm{~K}$, which is about $60 \mathrm{~K}$ below the desorption of the fluorophores. Since it is spectrally transparent, it will not emit and interfere with the emission from the aromatic molecules. As the layers percolate just before the desorption of 1-CH, it would impart thermal energy to the fluorophore. We report the decay constants of the excimers and traps by LIF for 2 $\mathrm{MeON}$ 2-MN and 2-EN alone as multilayer and bilayer with $1-\mathrm{CH}$.

\section{Experimental}

2-MN, 2-MeON, 2-EN, and 2-CH were purchased from commercial sources and their purity was checked by fluorescence. The samples were in turn placed in a stainless steel ampoule and outgassing was accomplished by freeze-pumpthaw cycles. Then by means of a leak valve (see Figure 1, (C)), 2-MN, 2-MeON, or 2-EN was vacuum deposited from the vapor onto a cryogenically cooled alumina surface $(M$ in Figure 1). Deposition was directed to the alumina crystal by a tube that extended the contents of the vapor as close as possible to the surface without impeding the rotation of the crystal. A second leak valve was used to deposit the 1-CH (K in Figure 1).

Referring to Figure 1, a super high pressure mercury lamp (H) was used in conjunction with a $0.25 \mathrm{~m}$ monochromator (G) that isolated appropriate emission lines for excitation of the substituted naphthalene molecules. The spectral emission was observed with a lens mounted within the vacuum chamber and fed through the high vacuum chamber port via a fiber optic cable (F) that was connected to the spectrometer (E). The spectra as a function of temperature were accumulated with an Ocean Optics (Dunedin, FL, USA) USB4000 spectrometer that recorded the spectrum every $400 \mathrm{~ms}$. Data was transferred to a MATLAB (MathWorks, Natick, MA, USA) file from which the wavelength-resolved TPD figures were made.

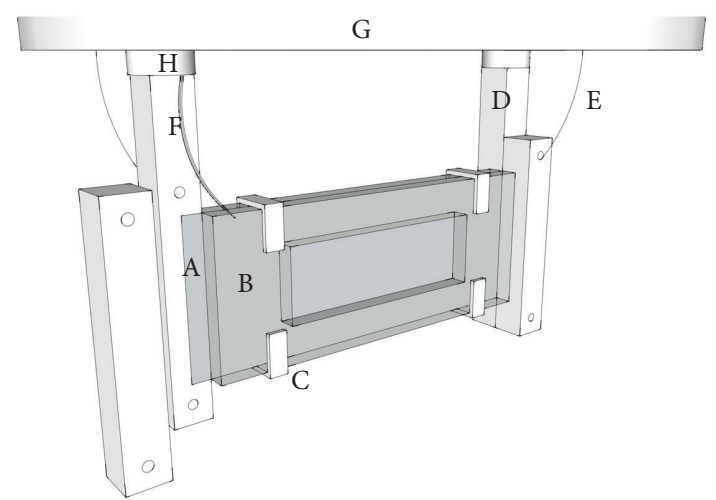

Figure 2: Details of the sample. A: tantalum foil, $\mathrm{B}: \mathrm{Al}_{2} \mathrm{O}_{3}$ (0001), $\mathrm{C}$ : molybdenum clips, D: copper posts, E: leads to power supply for ramping of temperature, F: chromel-alumel thermocouple, G: cryostat, H: sapphire spacers for thermal isolation.

For the LIF, a quadrupled Continuum (Santa Clara, CA, USA) Minilite Q-switched Nd: YAG laser was used (cf. A in Figure 1). For alignment purposes, a PC camera was used (L) to view the interior of the chamber. The fluorescence was detected by a combination of lenses that focused the light onto a $0.25 \mathrm{~m}$ monochromator (B in Figure 1). A high-speed amplifier took the signal to a very fast oscilloscope. The laser pulse was $5 \mathrm{~ns}$, and the instrumental response had a rate constant of more than $200 \times 10^{6} \mathrm{~s}^{-1}$. A National Instruments (Austin, TX, USA) LabVIEW program was written that took the average of 6 decay waveforms and then a least square curve-fitting program was used in which an exponential decay was assumed. The fit was visually monitored throughout the TPD experiment to ensure that the fit was good. The background waveform was subtracted each time a fluorescence decay was recorded.

For the TPD experiment, the temperature was linearly ramped at $2 \mathrm{~K} / \mathrm{s}$. Referring to Figure 2, a chromel-alumel thermocouple $(\mathrm{F})$ that was attached to the alumina crystal (B) sent a voltage to an analog-to-voltage converter box that was connected to a computer that controlled a power supply. This power supply fed current (E) through a tantalum foil (A) that was in thermal contact with the alumina via molybdenum clips (C). The crystal was mounted on two copper posts (D), through the sapphire spacers $(\mathrm{H})$ and unto the cryostat $(\mathrm{G})$.

The adlayer coverages were determined by using a diode laser that was directed at the surface during deposition [9]. The resulting optical interference was used to determine the rate of deposition and calibrated against the mass-specific quadrupole mass spectrometer (D in Figure 1) recording. The integrated mass spectral peaks were calibrated to the optical interference experiment and the coverages $(\Theta)$ are reported in monolayers (ML) with an error of $\pm 25 \%$. The activation energy for desorption, $E_{a}$, was calculated by Redhead analysis by assuming a first-order desorption kinetics as described by King and is based on the mass spectral peak desorption temperature, $T_{p}$ [10-12]. (cf. Table 1). The uncertainties in the desorption temperatures and the propagated error in the activation energies are $\pm 3 \%$. 
TABLE 1: Peak desorption temperatures, activation energies of desorption, and LIF excimer and trap decay rate constants for multilayer and bilayer (with 1-CH) for the two component decay.

\begin{tabular}{|c|c|c|c|}
\hline & $2-\mathrm{MeON}$ & $2-\mathrm{MN}$ & $2-\mathrm{EN}$ \\
\hline$T_{P}( \pm 3 \%)(\mathrm{K})$ & 275 & 264 & 268 \\
\hline$E_{a}( \pm 3 \%)(\mathrm{kJ} / \mathrm{mol})$ & 72 & 69 & 70 \\
\hline \multicolumn{4}{|l|}{ Rate constant $( \pm 20 \%)\left(\times 10^{6} \mathrm{~s}^{-1}\right)$} \\
\hline \multicolumn{4}{|l|}{ Multilayer } \\
\hline \multicolumn{4}{|l|}{ (i) Excimer } \\
\hline Long component & 2 & 6 & 5 \\
\hline Short component & 35 & 25 & 23 \\
\hline (ii) Trap & 100 & 44 & 23 \\
\hline \multicolumn{4}{|l|}{ Bilayer } \\
\hline \multicolumn{4}{|l|}{ (i) Excimer } \\
\hline Long component & 2 & 4 & 7 \\
\hline $\begin{array}{l}\text { Short component } \\
\text { (second excimer) }\end{array}$ & $30(36)$ & $25(45)$ & $23(42)$ \\
\hline (ii) Trap (second trap) & $67(95)$ & $18(33)$ & $43(57)$ \\
\hline
\end{tabular}

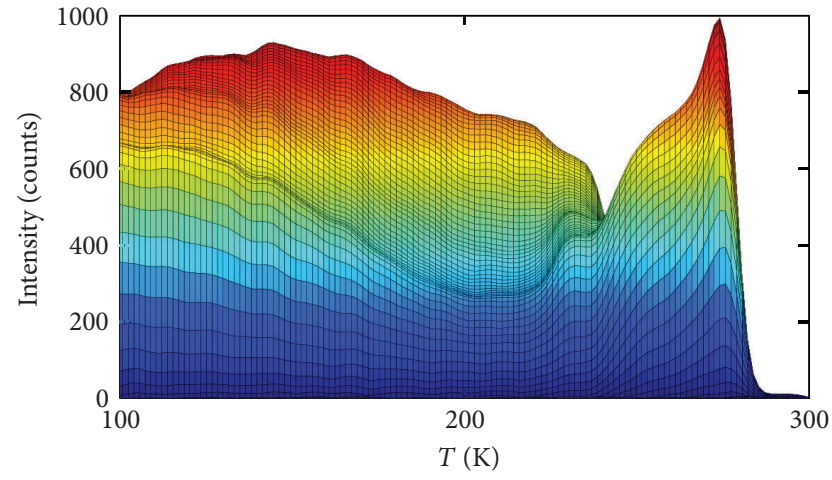

FIgURE 3: Excimer fluorescence TPD of 2-MeON. $\Theta_{2-\mathrm{MeON}} \sim$ $240 \mathrm{ML}$.

\section{Results and Discussion}

3.1. 2-MeON Multilayer and 2-MeON : 2-CH Bilayer. Shown in Figure 3 is the excimer fluorescence as a function of temperature for multilayers of $2-\mathrm{MeON}$. The excimer intensity at $\lambda_{\max } \sim 410 \mathrm{~nm}$ decreases slightly during the TPD presumably due to thermal quenching. At around $238 \mathrm{~K}$, the adlayer undergoes a disorder-to-order transition, and the intensity of the excimer with $\lambda_{\max } \sim 423 \mathrm{~nm}$ decreases as the intensity from what will be termed trap at $\lambda_{\max } \sim 368 \mathrm{~nm}$ increases to a maximum at $274 \mathrm{~K}$ before desorption at $275 \mathrm{~K}$ with an activation energy of $72 \mathrm{~kJ} / \mathrm{mol}$. The red shift is due to the relaxation of the adlayer to a more stable arrangement that results in excimers with lower energy [2]. Shown in Figure 4 is the emission intensity of $2-\mathrm{MeON}$ as a function of temperature in which 1-CH was deposited as a separate layer on top of 2-MeON.

The decay due to relaxation via fluorescence of multilayer 2-MeON exhibited two slopes in the natural log of the waveform. The decay constants of these two components are shown in Figure 5. The fast component is relatively constant over the entire TPD. The temperature averages of the two

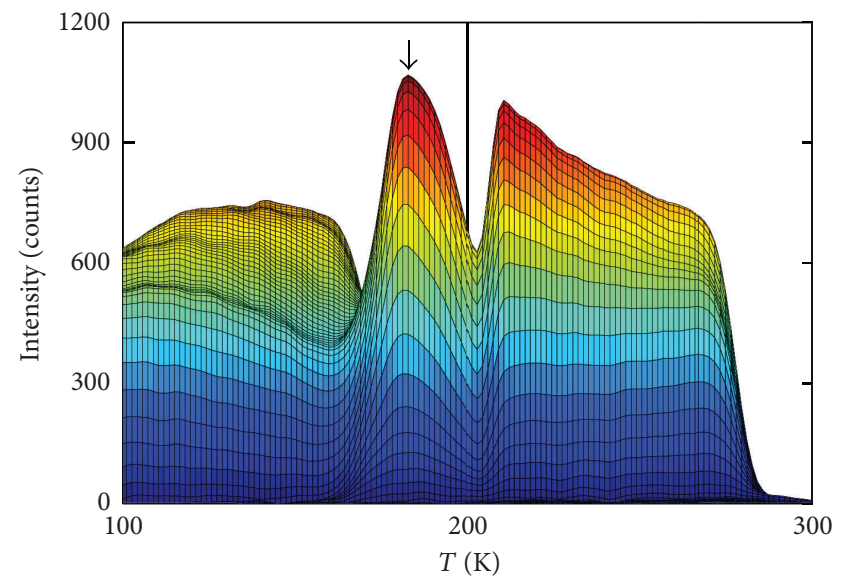

FIGURE 4: Excimer fluorescence TPD of 2-MeON $\left(\Theta_{2-\mathrm{MeON}} \sim\right.$ $190 \mathrm{ML})$ in which 1-CH was deposited as a second layer $\left(\Theta_{1-\mathrm{CH}} \sim\right.$ $300 \mathrm{ML}$ ). Vertical grid at $200 \mathrm{~K}$ points to the temperature at which 1-CH desorbs. Arrow points to formation of clusters.

decay rate constants are recorded in Table 1. Also shown in Table 1 is the rate constant of the trap observed at $\lambda_{\max } \sim$ 368.

For the bilayer at the deposition temperature, the rate constants for the short and long components are the same as in the multilayer within experimental error (cf. Figure 5). However, as the surface is heated, 1-CH has a definite effect on the rate constants of both fast and slow components. As 1-CH gains thermal energy during the TPD, 2-MeON is affected by the collisional energy transfer with $1-\mathrm{CH}$, and at $185 \mathrm{~K}$, the intensity of the excimer decreases while the trap intensity reaches a maximum. (cf. arrow in Figures 4 and 5). At this point in the TPD, a reasonable assumption is that the $1-\mathrm{CH}$ has percolated into the $2-\mathrm{MeON}$ layer, thereby forming clusters similar to those observed in other molecules $[9,13]$. The result is the shift in the spectrum to $\lambda_{\max } \sim$ $364 \mathrm{~nm}$, such as that observed in solution, and the trap rate 


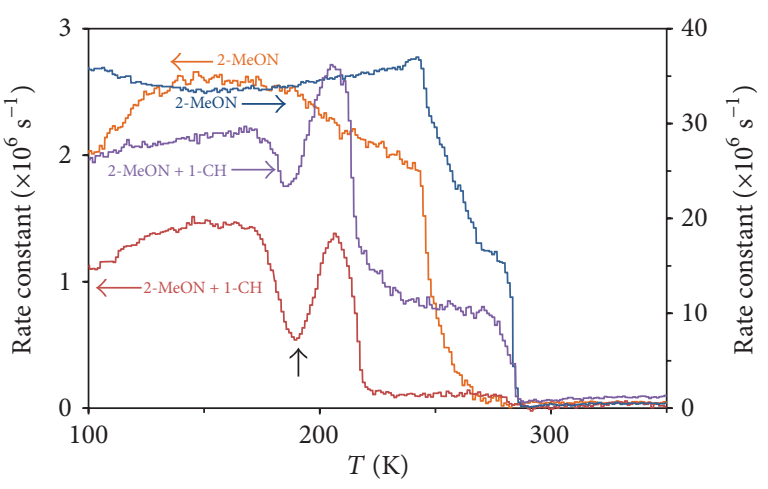

FIGURE 5: LIF decay rate constants of 2-MeON excimer as a function of temperature. 2-MeON: rate constants of 2-MeON multilayer. Scale to left for slower $\left(\Theta_{2-\mathrm{MeON}} \sim 360 \mathrm{ML}\right)$ and to the right for faster component $\left(\Theta_{2-\mathrm{MeON}} \sim 490 \mathrm{ML}\right) .2-\mathrm{MeON}+1-\mathrm{CH}$ : rate constants of $2-\mathrm{MeON}$ when $2-\mathrm{MeON}$ and $1-\mathrm{CH}$ were prepared as a bilayer. Slower component scale on left $\left(\Theta_{2-\mathrm{MeON}} \sim 260 \mathrm{ML}, \Theta_{1-\mathrm{CH}} \sim\right.$ $380 \mathrm{ML})$, faster component scale on right $\left(\Theta_{2-\mathrm{MeON}} \sim 170 \mathrm{ML}\right.$, $\left.\Theta_{1-\mathrm{CH}} \sim 220 \mathrm{ML}\right)$. Arrow points to the formation of clusters.

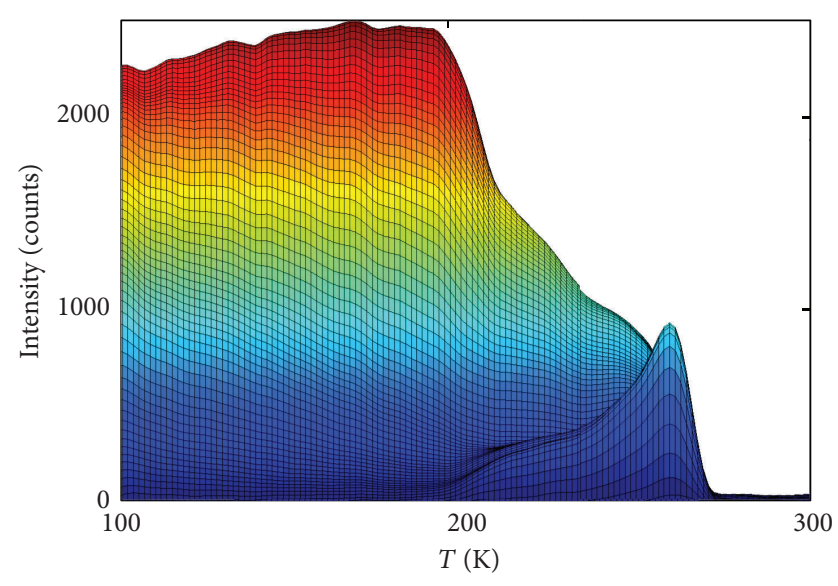

FIGURE 6: Excimer fluorescence TPD of 2-MN. $\Theta_{2-\mathrm{MN}} \sim 120 \mathrm{ML}$.

constants reported in Table 1 reflect this model. Here, both components of the LIF excimer decay constants decrease (cf. arrow in Figure 5). Subsequent to the desorption of 1-CH at $200 \mathrm{~K}\left(E_{a} \sim 52 \mathrm{~kJ} / \mathrm{mol}\right)$, the fast component increases to a maximum (the temperature at which 1-CH desorbs is noted by the vertical grid line at $200 \mathrm{~K}$ ). In the bilayer, the excimer that forms after the desorption of 1-CH can be expected to be altered, and the observed difference in the decay constants of about $20 \%$ corroborate this tentative model (Table 1 ).

For the bilayer, the traps were observed at two temperatures: at $185 \mathrm{~K}$ where the first maximum in intensity was observed. Here, the rate constant is the first entry in Table 1, and the second trap intensity was observed at $210-269 \mathrm{~K}$, where the rate constant is the second entry. From a comparison of the rate constants for the traps in the multilayer and bilayer, the close agreement would lead to the conclusion that the second trap that occurs after the 1-CH has desorbed is similar to the multilayer trap.

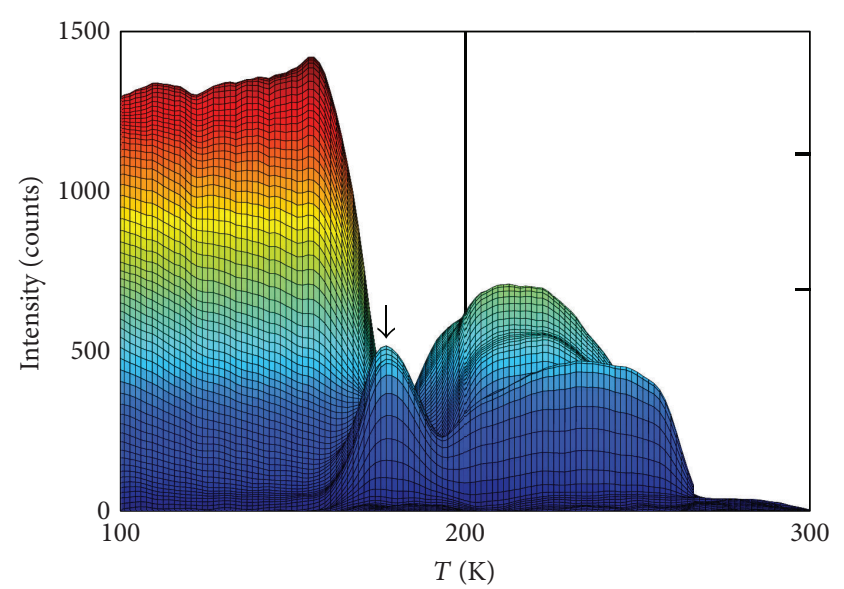

FIgURE 7: Excimer fluorescence TPD of 2-MN $\left(\Theta_{2-\mathrm{MN}} \sim 35 \mathrm{ML}\right)$ in which 1-CH was deposited as a second layer $\left(\Theta_{1-\mathrm{CH}} \sim 240 \mathrm{ML}\right)$. Grid line at $200 \mathrm{~K}$ is the temperature at which $1-\mathrm{CH}$ desorbs. Arrow points to the formation of clusters.

3.2. $2-M N$ Multilayer and 2-MN:1-CH Bilayer. Figure 6 is a plot of the fluorescence intensity of 2-MN multilayer on alumina as a function of temperature. The excimer intensity $\lambda_{\max } \sim 417 \mathrm{~nm}$ decreases at $200 \mathrm{~K}$ that signals the onset of the ordering transition, as evidenced by the trap emission that increases to a maximum at $260 \mathrm{~K}$ with $\lambda_{\max } \sim 358 \mathrm{~nm}$. Desorption occurs at $264 \mathrm{~K}$. Although not shown, the longer component of the LIF decay for the excimer remains relatively constant as does the fluorescence intensity, as indicated by the wavelength-resolved TPD in Figure 6. At the ordering transition, the rate decreases by almost $35 \%$.

The inclusion of the 1-CH as a bilayer to 1-MN significantly changes the surface dynamics, as seen from the fluorescence intensity profile as a function of temperature in Figure 7 when compared to Figure 6 (the temperature at which 1-CH desorbs is noted by the vertical grid line at $200 \mathrm{~K}$ ) the first maximum in the trap emission occurs at $185 \mathrm{~K}$ with a $\lambda_{\max } \sim 356 \mathrm{~nm}$; the second maximum occurs at $240 \mathrm{~K}$ with a $\lambda_{\max } \sim 358 \mathrm{~nm}$. As in the $2-\mathrm{MeON}$ bilayer, the first maximum in the trap intensity is presumably associated with the $2-\mathrm{MN} / 1-\mathrm{CH}$ cluster, since $1-\mathrm{CH}$ molecules would have sufficient thermal energy to percolate into the adlayer containing $2-\mathrm{MN}$ and effectively solvate the fluorophore. Upon desorption of the 1-CH (grid line in Figure 7), the excimer formation can again resume.

The two components of the fluorescence decay during the TPD are shown in Figure 8. The rate constants are similar to that of the corresponding components in $2-\mathrm{MeON}$ and remain relatively constant until the ordering transition, at which point, the rate of the slow component decreases by about $20 \%$. The rate constant of the trap is seen in Table 1 . Although not shown, the profile of the rate constant taken with $\lambda_{\max } \sim 358 \mathrm{~nm}$ of the trap fluorescence exactly reflects the cross-sectional plot of the wavelength-resolved TPD of Figure 6.

In the bilayer, the dip in the rate constant seen in Figure 7 occurs in both the long and short excimer components, but for different reasons: for the long component, the dip is 


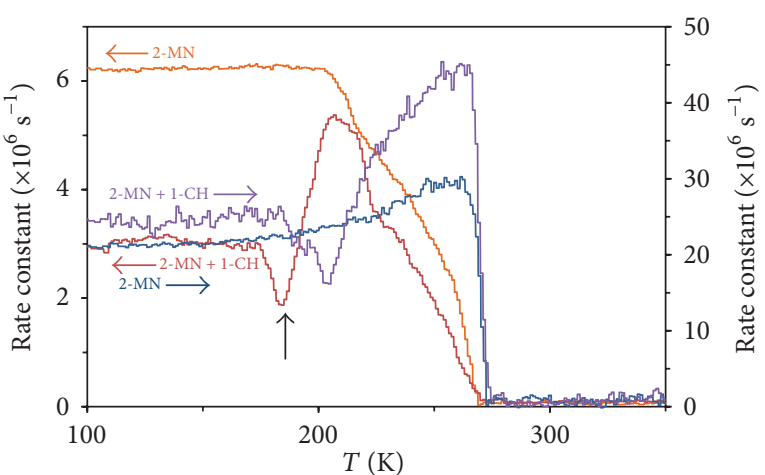

FIGURE 8: LIF decay rate constants of 2-MN excimer as a function of temperature. 2-MN: rate constants of 2-MN multilayer. Scale to left for slower $\left(\Theta_{2-\mathrm{MN}} \sim 59 \mathrm{ML}\right)$ and to the right for faster component $\left(\Theta_{2-\mathrm{MN}} \sim 54 \mathrm{ML}\right) .2-\mathrm{MN}+1-\mathrm{CH}$ : rate constants of 2-MN when 2$\mathrm{MN}$ and 1-CH were prepared as a bilayer. Slower component scale on left $\left(\Theta_{2-\mathrm{MN}} \sim 21 \mathrm{ML}, \Theta_{1-\mathrm{CH}} \sim 260 \mathrm{ML}\right)$, faster component scale on right $\left(\Theta_{2-\mathrm{MN}} \sim 100 \mathrm{ML}, \Theta_{1-\mathrm{CH}} \sim 370 \mathrm{ML}\right)$. Arrow points to the formation of clusters.

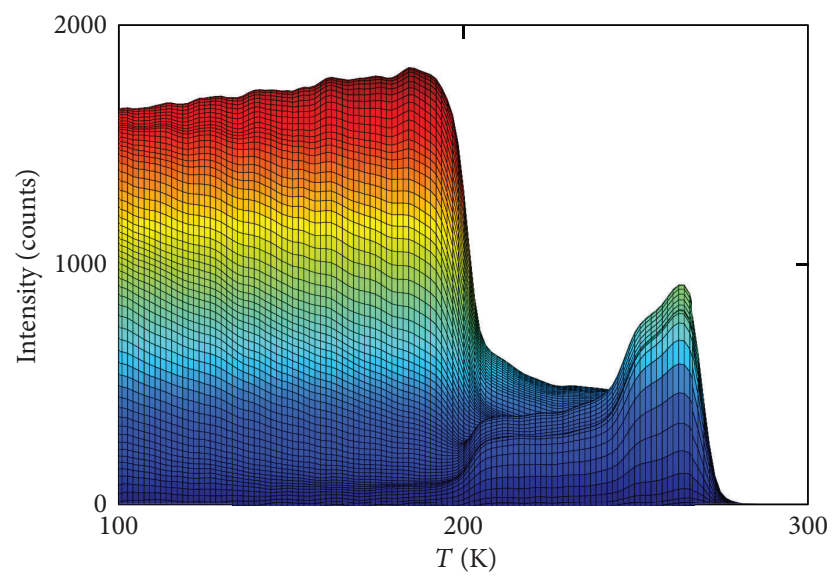

FIGURE 9: Excimer fluorescence TPD of multilayer 2-EN $\left(\Theta_{2-\mathrm{EN}} \sim\right.$ $87 \mathrm{ML})$.

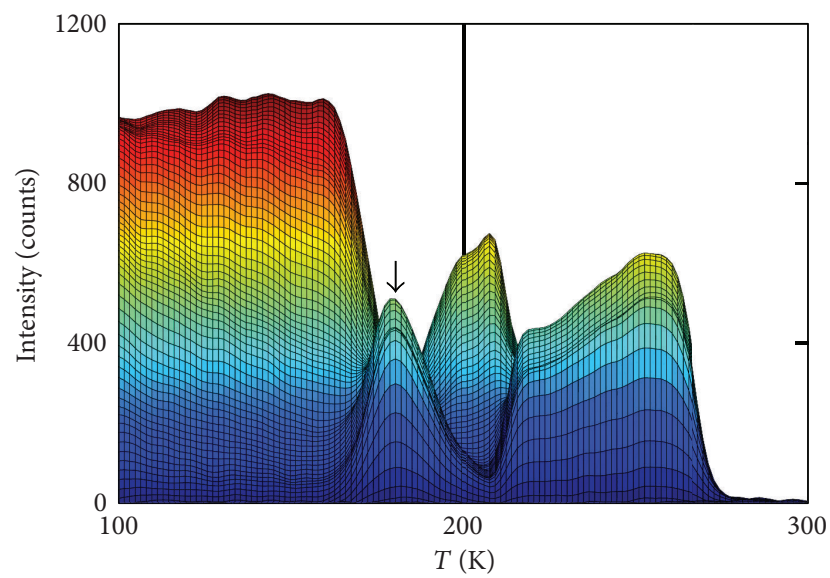

FIGURE 10: Excimer fluorescence TPD of bilayer of 2-EN $\left(\Theta_{2 \text {-EN }} \sim\right.$ $64 \mathrm{ML}): 1-\mathrm{CH}\left(\Theta_{1-\mathrm{CH}} \sim 280 \mathrm{ML}\right)$ bilayer. Arrow points to the formation of clusters.

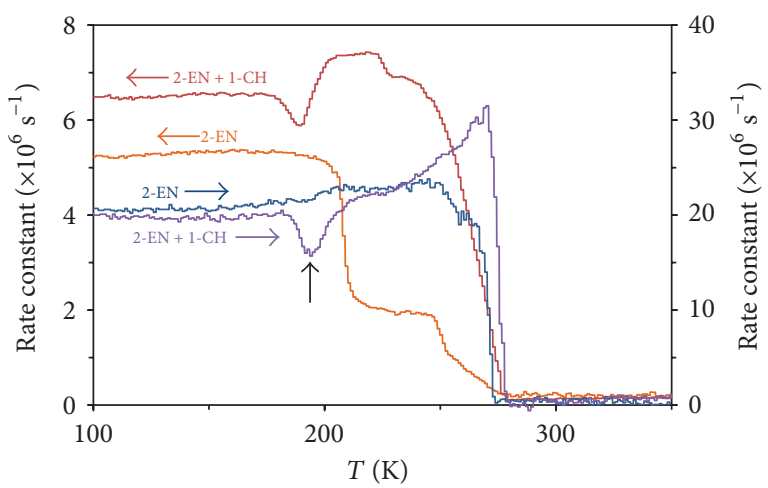

FIGURE 11: LIF decay rate constants of 2-EN excimer as a function of temperature. 2-EN: rate constants of 2-EN multilayer. Scale to left for slower $\left(\Theta_{2-\mathrm{EN}} \sim 130 \mathrm{ML}\right)$ and to the right for faster component $\left(\Theta_{2-\mathrm{EN}} \sim 380 \mathrm{ML}\right) .2$-EN + 1-CH: rate constants of 2-EN when 2-EN and 1-CH were prepared as a bilayer. Slower component scale on left $\left(\Theta_{2-\mathrm{EN}} \sim 220 \mathrm{ML}, \Theta_{1-\mathrm{CH}} \sim 310 \mathrm{ML}\right)$, faster component scale on right $\left(\Theta_{2-\mathrm{EN}} \sim 340 \mathrm{ML}, \Theta_{1-\mathrm{CH}} \sim 260 \mathrm{ML}\right)$. Arrow points to the formation of clusters.

coincident with the formation of the presumed 2-MN : 1-CH cluster, and the dip in the short component is associated with the desorption of 1-CH.

For the trap, there are two rate constants. The first entry in Table 1 is associated with the relaxation of the clustered 2-MN molecule that should closely resemble the rate in solution. The second entry is the trap that also occurs in the multilayer and the rates are similar.

As was the case for 2-MeON, differences in the excimer types in the aftermath of the desorption of 1-CH are reflected in the rates. In this case as well as in the $2-\mathrm{MeON}$, the rates are larger for the bilayer.

3.3. 2-EN Multilayer and 2-EN:1-CH Bilayer. In Table 1, the peak desorption temperature and activation energy for 2-EN on alumina are given, as well as the rate constants for the multilayer and bilayer with 1-CH. The fluorescence intensities of the multilayer and bilayer as a function of temperature are shown in Figures 9 and 10, respectively. The rate constants as a function of temperature for the respective molecules are shown in Figure 11. The rate for the long component decreases rapidly to about $30 \%$ at the disorderto-order transition that occurs at $210 \mathrm{~K}$, whereas the fast component remains relatively constant through the TPD.

For the bilayer, the presumed cluster forms at $186 \mathrm{~K}$ with the molecular fluorescence at $\lambda_{\max } \sim 356 \mathrm{~nm}$. The excimer that resumes has a $\lambda_{\max } \sim 423$ and for the short component is considerably higher rate than the corresponding rate for the multilayer as seen in Figure 11. The disruption in the formation of excimer is clearly visible, where minima in the rate constants for both long and short components are observed (Figure 11).

In summary, we have reported here, the LIF decay rate constants for 2-MeON, 2-MN, and 2-EN on an alumina surface that are consistent with previously reported rates for similarly substituted naphthalenes [3-7]. The decay has two 
components: the slower component for the three molecules are $4 \pm 3 \times 10^{6} \mathrm{~s}^{-1}$. The faster component for $2-\mathrm{MN}$ and 2 -EN is about $24 \pm 4 \times 10^{6} \mathrm{~s}^{-1}$ and almost $50 \%$ higher for $2-\mathrm{MeON}$.

\section{Acknowledgment}

The authors are grateful for the financial support from their respective institutions in the form of student stipends for the summer research program.

\section{References}

[1] H. Nakayama, T. Hosokawa, and K. Ishii, "Fluorescence spectra and energy transfer in amorphous naphthalene," Chemical Physics Letters, vol. 289, no. 3-4, pp. 275-280, 1998.

[2] J. B. Birks, Photophysics of Aromatic Molecules, Wiley-Interscience, London, UK, 1970.

[3] J. T. Aldekomo and J. B. Birks, "Excimer' fluorescence. VII. Spectral studies of naphthalene and its derivatives," Proceedings of the Royal Society of London A, vol. 284, no. 1399, pp. 551-565, 1965.

[4] T. Azumi and H. Azumi, "Lifetimes of excimer fluorescence of naphthalene," Bulletin of the Chemical Society of Japan, vol. 39, no. 11, pp. 2317-2320, 1966.

[5] F. Li, J. Lee, and E. R. Bernstein, "Spectroscopy of naphthalene in simple molecular liquids," Journal of Physical Chemistry, vol. 87, no. 7, pp. 1175-1180, 1983.

[6] R. B. Cundall and L. C. Pereira, "Temperature effects on the relaxation parameters of the 1-methylnaphthalene excimer," Chemical Physics Letters, vol. 15, no. 3, pp. 383-386, 1972.

[7] Birks J. B. and T. A. King, "Excimer fluorescence VIII. Lifetimes studies of 1,6-dimethylnaphthalene," Proceedings of the Royal Society of London A, vol. 291, no. 1425, pp. 244-256, 1966.

[8] C. L. Binkley, T. C. Judkins, N. C. Freyshlag, K. A. Martin, and A. M. Nishimura, "Steric effect of methyl, methoxy, and ethyl substituents on the excimer formation of naphthalene on $\mathrm{Al}_{2} \mathrm{O}_{3}(0001)$," Surface Science, vol. 603, no. 14, pp. 2207-2209, 2009.

[9] B. J. Haddock, S. L. Cowell, L. Meiling, K. A. Martin, and A. M. Nishimura, "Formation of molecular clusters by percolation of water through p-bromochlorobenzene adlayer on $\mathrm{Al}_{2} \mathrm{O}_{3}(0001)$," Surface Science, vol. 569, no. 1-3, pp. 56-62, 2004.

[10] P. A. Redhead, “Thermal desorption of gases," Vacuum, vol. 12, no. 4, pp. 203-211, 1962.

[11] F. M. Lord and J. S. Kittelberger, "On the determination of activation energies in thermal desorption experiments," Surface Science, vol. 43, no. 1, pp. 173-182, 1974.

[12] D. A. King, "Thermal desorption from metal surfaces: a review," Surface Science, vol. 47, no. 1, pp. 384-402, 1975.

[13] M. A. Evans, D. R. Hoss, K. E. Howard et al., "Use of fluorescence to probe the surface dynamics during disorder-to-order transition and cluster formation in dihalonaphthalene-water thin films on $\mathrm{Al}_{2} \mathrm{O}_{3}(0001)$," Thin Solid Films, vol. 515, no. 4, pp. 1370-1376, 2006. 

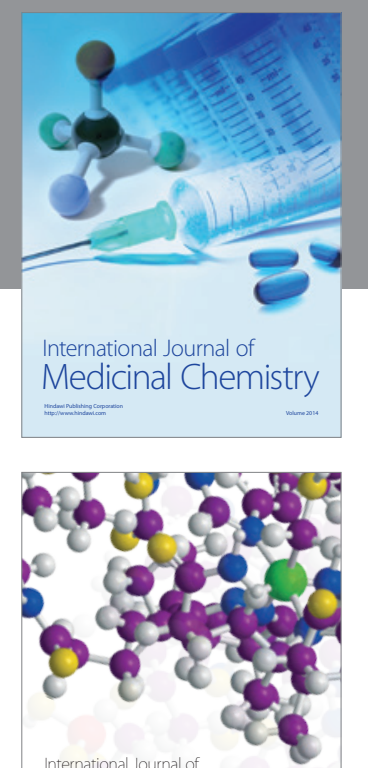

\section{Carbohydrate} Chemistry

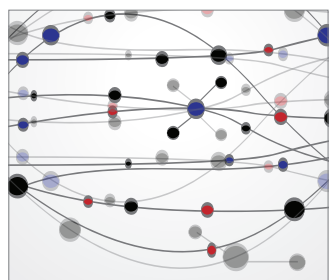

The Scientific World Journal
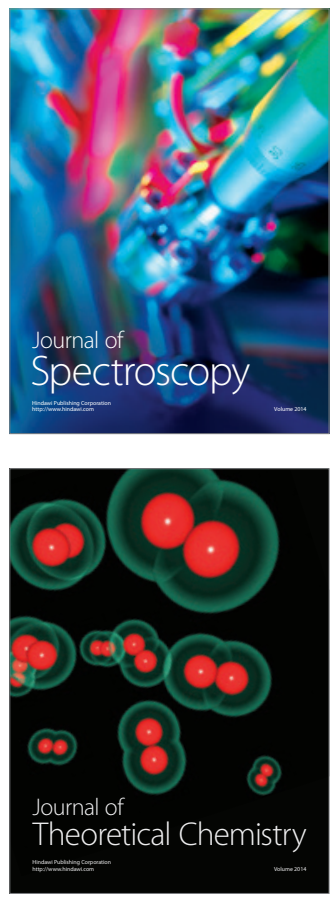
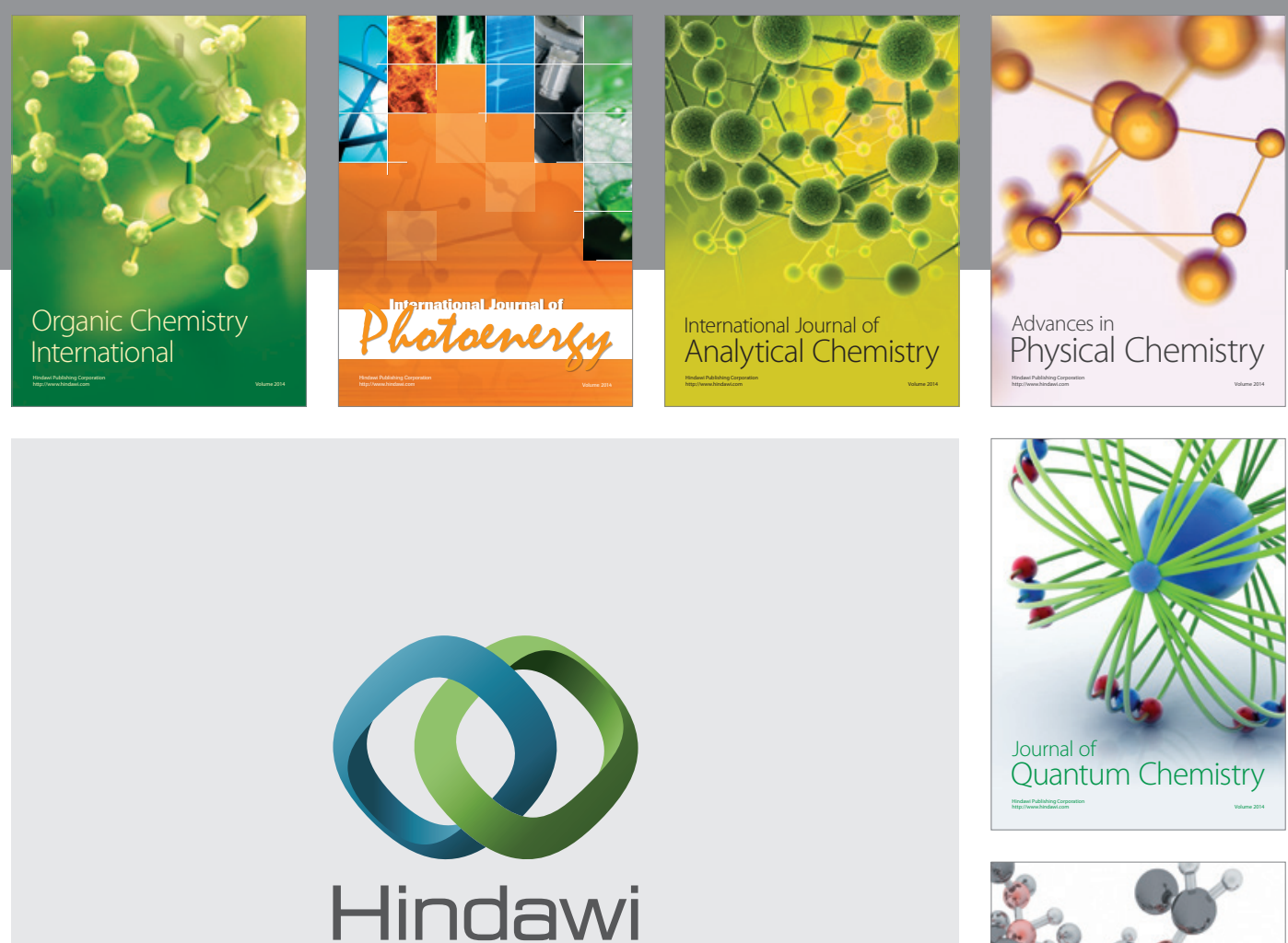

Submit your manuscripts at

http://www.hindawi.com

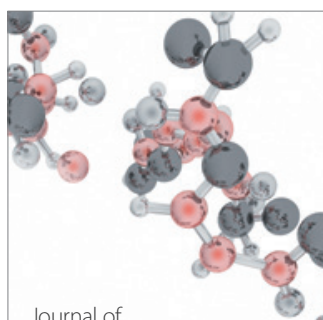

Analytical Methods

in Chemistry

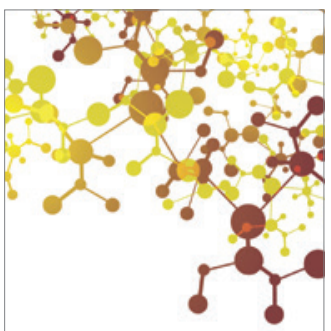

Journal of

Applied Chemistry

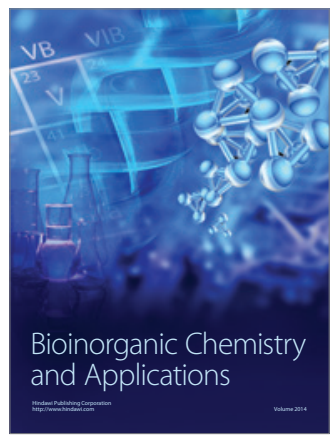

Inorganic Chemistry
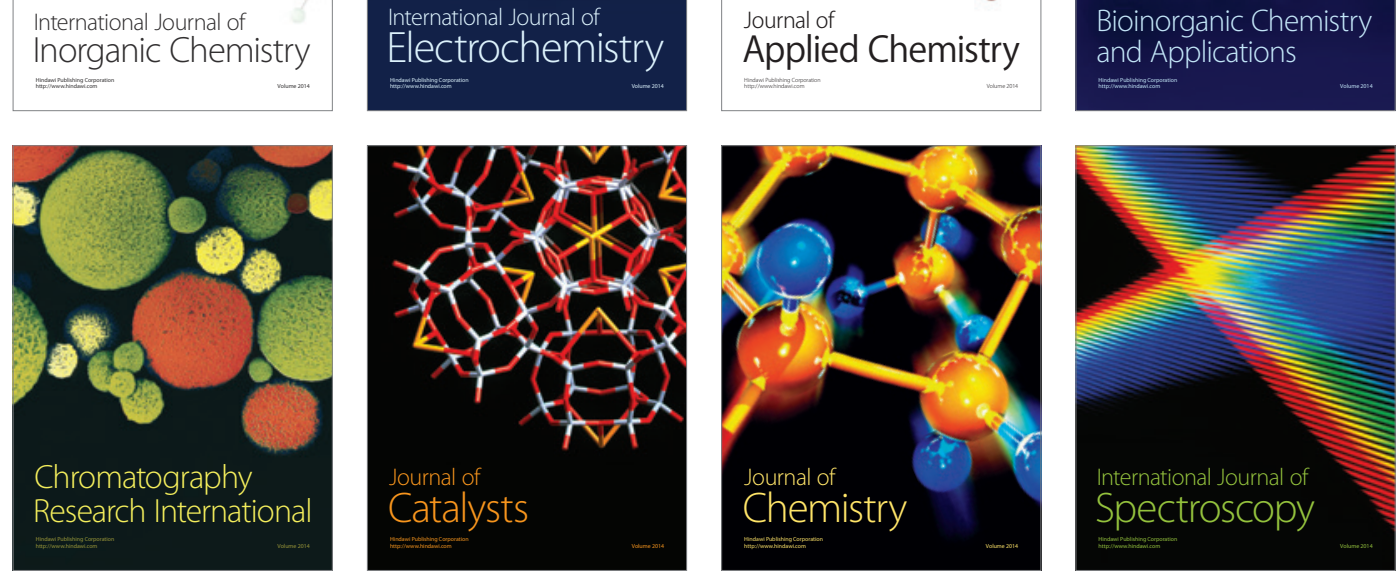